\title{
Stage I Intrahepatic Bile Duct Cancer AJCC v8
}

National Cancer Institute

\section{Source}

National Cancer Institute. Stage I Intrahepatic Bile Duct Cancer A/CC v8. NCI Thesaurus. Code C134610.

Stage I includes: IA (T1a, N0, M0); IB (T1b, N0, M0). T1a: Solitary tumor equal to or less than $5 \mathrm{~cm}$ without vascular invasion. T1 b: Solitary tumor larger than $5 \mathrm{~cm}$ without vascular invasion. N0: No regional lymph node metastasis. M0: No distant metastasis. (AJCC 8th ed.) 